\title{
Entanglement generation in continuously coupled parametric generators
}

\author{
Jiří Herec, ${ }^{1}$ Jaromír Fiurášek, ${ }^{1,2}$ and Ladislav Mišta Jr. ${ }^{1}$ \\ ${ }^{1}$ Department of Optics, Palacký University, 17. listopadu 50, 77200 Olomouc, Czech Republic \\ ${ }^{2}$ Ecole Polytechnique, CP 165/59, Université Libre de Bruxelles, 1050 Bruxelles, Belgium
}

(Dated: October 30, 2018)

\begin{abstract}
We investigate a compact source of entanglement. This device is composed of a pair of linearly coupled nonlinear waveguides operating by means of degenerate parametric downconversion. For the vacuum state at the input the generalized squeeze variance and logarithmic negativity are used to quantify the amount of nonclassicality and entanglement of output beams. Squeezing and entanglement generation for various dynamical regimes of the device are discussed.
\end{abstract}

\section{INTRODUCTION}

Quantum entanglement and its consequences puzzled physicists since the famous paper by Einstein, Podolsky and Rosen [1]. It was recognized in recent years that the entanglement is not only an intriguing and mind boggling feature of quantum mechanics but also a crucial and extremely useful resource for information processing. The various protocols relying on the entanglement include quantum teleportation [2], entanglement swapping [3] , dense coding [4], quantum cryptography [ [] $]$ and quantum computing [6]. The efficiency of quantum information processing significantly depends on the degree of entanglement of the quantum state shared by the parties involved in a given protocol. It is therefore highly desirable to establish reliable sources of pure strongly entangled states.

Quantum optics provides a natural and convenient framework for the generation of entangled states, their manipulation and measurement. The above-mentioned protocols have been originally established for the quantum systems with two-dimensional Hilbert spaces - the qubits. The polarization entangled pairs of photons generated by means of the spontaneous parametric downconversion proved to be a very good source of entangled qubits and they were employed in a large number of experimental demonstrations of quantum information processing.

Recently, however, a significant attention has been paid to the quantum information processing (QIP) with systems whose Hilbert space is infinite dimensional. A typical example of such a system is a single mode of the optical field whose Hilbert space is spanned by the infinite (but countable) number of the Fock states $|n\rangle$. It is in the spirit of this latter approach that one considers the quadrature operators $\hat{x}, \hat{p}$ and one speaks about QIP with continuous variables (CV). Similarly as in the case of qubits, the parametric downconversion provides a source of CV entanglement. Here, the relevant entangled state is the so-called two-mode squeezed vacuum (sometimes also called the twin beam) that can be either prepared by nondegenerate downconversion or via mixing of two single-mode squeezed states on a balanced beam splitter. This last-mentioned approach has been in fact used in the experiment on the teleportation of continuous variables
7]. The whole setup for the generation of the CV entangled state is certainly nontrivial and quite complicated. A natural question arises whether it could be possible to integrate the generation of the single mode squeezed states and the mixing on a beam splitter into a single small and compact device that would serve as a source of the entanglement.

In this paper we show that the nonlinear optical couplers that have been thoroughly investigated during recent years both theoretically and experimentally (see [8, 9] and references therein) can serve exactly for that purpose. We theoretically investigate the generation of squeezing and entanglement in the nonlinear optical coupler consisting of a pair of nonlinear waveguides operating by means of the degenerate parametric downconversion. The waveguides are placed close together so that the two modes can overlap and exchange energy via linear coupling. We study the dependence of the generalized squeezing as well as CV entanglement on the coupler's parameters. The entanglement is quantified by the logarithmic negativity [10], which is an easily computable entanglement monotone. Finally, the optimum phase matching condition that yields the maximal entanglement is analyzed.

\section{THE MODEL AND THE EQUATIONS OF MOTION}

The device under study is schematically depicted in Fig. 1. The nonlinear coupler consists of two linearly coupled waveguides where the degenerate parametric

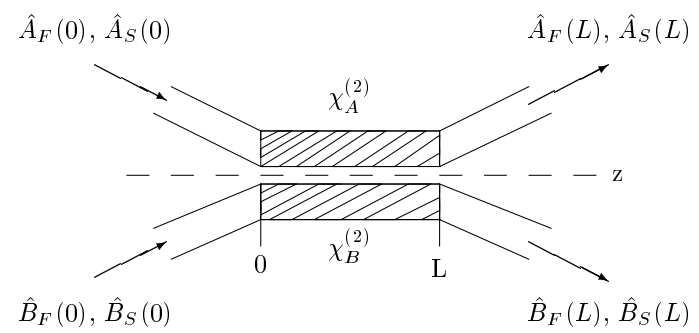

FIG. 1: Sketch of nonlinear coupler formed from two nonlinear waveguides $A$ and $B$ with susceptibilities $\chi^{(2)}$. 
downconversion takes place. A 'blue' photon with frequency $2 \omega$ may split into a pair of identical 'red' photons and also an inverse process may occur. Assuming a strong coherent pumping of the modes with frequency $2 \omega$ (second-harmonic modes) and a linear coupling between the modes with frequency $\omega$ (fundamental modes) in the lossless and nondispersive media, we can describe such a device by the following effective interaction momentum operator 11]

$$
\hat{G}_{\mathrm{int}}=\hbar\left(g_{A}^{*} \hat{A}_{F}^{2}+g_{B}^{*} \hat{B}_{F}^{2}+2 g_{L} \hat{A}_{F} \hat{B}_{F}^{\dagger}+\text { h.c. }\right),
$$

where $\hat{A}_{F}\left(\hat{A}_{F}^{\dagger}\right)$ is annihilation (creation) operator of the fundamental mode in the first waveguide and $\hat{B}_{F}\left(\hat{B}_{F}^{\dagger}\right)$ is annihilation (creation) operator of the fundamental mode in the second waveguide. The constant $g_{A}\left(g_{B}\right)$ is a product of the strong coherent amplitude of the secondharmonic mode in the first (second) waveguide and the nonlinear coupling constant, which is proportional to the quadratic susceptibility $\chi_{A}^{(2)}\left(\chi_{B}^{(2)}\right)$. The constants $g_{A}$ and $g_{B}$ determine the efficiency of the downconversion processes. Linear coupling constant denoted as $g_{L}$ describes the energy exchange between the waveguides by means of coupling via the evanescent waves. The symbol $\hbar$ denotes the reduced Planck constant and h.c. represents Hermitian conjugate terms.

We shall work in the interaction picture where the spatial evolution of an operator $\hat{O}$ is governed by the Heisenberg-like equation (see [8, [9] and references therein)

$$
i \hbar \frac{d \hat{O}}{d z}=\left[\hat{G}_{\text {int }}, \hat{O}\right] .
$$

Here $z$ is a spatial coordinate along the direction of propagation, $\hat{G}_{\text {int }}$ is an interaction momentum operator and [,] denotes a commutator. Using the momentum operator (11), we obtain the Heisenberg equations of motion in the interaction picture of the form:

$$
\begin{aligned}
& \frac{d \hat{A}_{F}}{d z}=2 i g_{A} \hat{A}_{F}^{\dagger}+2 i g_{L}^{*} \hat{B}_{F}, \\
& \frac{d \hat{B}_{F}}{d z}=2 i g_{B} \hat{B}_{F}^{\dagger}+2 i g_{L} \hat{A}_{F} .
\end{aligned}
$$

For our purposes it is convenient to deal with the quadrature components $\hat{x}, \hat{p}$ rather than with the creation and annihilation operators. The quadratures can be directly measured via balanced homodyne detection. Recall that the quadrature components can be expressed as linear combinations of the creation and annihilation operators as follows:

$$
\begin{array}{ll}
\hat{x}_{A}=\frac{\sqrt{2}}{2}\left(\hat{A}_{F}^{\dagger}+\hat{A}_{F}\right), & \hat{p}_{A}=i \frac{\sqrt{2}}{2}\left(\hat{A}_{F}^{\dagger}-\hat{A}_{F}\right), \\
\hat{x}_{B}=\frac{\sqrt{2}}{2}\left(\hat{B}_{F}^{\dagger}+\hat{B}_{F}\right), & \hat{p}_{B}=i \frac{\sqrt{2}}{2}\left(\hat{B}_{F}^{\dagger}-\hat{B}_{F}\right) .
\end{array}
$$

We further express the (generally complex) coupling constants in terms of their amplitudes and phases,

$$
g_{j}=\left|g_{j}\right| \exp \left(i \varphi_{j}\right) \quad \text { for } \quad j=L, A, B .
$$

With these definitions at hand, we can derive from Eq. (3) the Heisenberg equations for the quadrature operators. We write them down in the compact matrix form,

$$
\frac{d \hat{\xi}}{d z}=\mathbf{M} \hat{\xi}
$$

where

$$
\mathbf{M}=\left(\begin{array}{cccc}
-S_{A} & C_{A} & S_{L} & -C_{L} \\
C_{A} & S_{A} & C_{L} & S_{L} \\
-S_{L} & -C_{L} & -S_{B} & C_{B} \\
C_{L} & -S_{L} & C_{B} & S_{B}
\end{array}\right), \quad \hat{\xi}=\left(\begin{array}{c}
\hat{x}_{A} \\
\hat{p}_{A} \\
\hat{x}_{B} \\
\hat{p}_{B}
\end{array}\right)
$$

and

$$
S_{j}=2\left|g_{j}\right| \sin \left(\varphi_{j}\right), \quad C_{j}=2\left|g_{j}\right| \cos \left(\varphi_{j}\right) .
$$

Eq. (6) represents a system of linear differential equations with constant coefficients that can be solved very simply by determining the eigenvalues and eigenvectors of the matrix M. Explicit analytical expressions for the solution can be found in [11]. Here we write the solution in a formal matrix notation

$$
\hat{\xi}(z)=\mathbf{S}(z) \hat{\xi}(0), \quad \mathbf{S}(z)=e^{\mathbf{M} z} .
$$

This evolution represents a linear canonical transformation of the quadrature operators. All possible linear canonical transformations of $N$ modes form a group of symplectic (also called Bogolyubov) transformations $S p(2 N, R)$. In our case, for each $z$ the matrix $\mathbf{S}(z)$ is an element from the four dimensional representation of the symplectic group $S p(4, R)$.

One of the most important features of the symplectic transformations is that they transform Gaussian states again onto Gaussian states. The Gaussian states are those whose Wigner function is a Gaussian. Such states are thus fully characterized by the first and second moments of the quadrature operators. Moreover, the first moments (mean values) of the quadratures can be always set to zero via appropriate displacements applied locally to each mode. Since we are interested in entanglement properties of the state generated in the coupler, we can focus solely on the evolution of the second moments.

\section{THE GENERALIZED SQUEEZE VARIANCE}

It is convenient to arrange the second moments into the covariance matrix $\mathbf{V}$ whose elements are defined as follows [12]:

$$
V_{j k}=\frac{1}{2}\left(\left\langle\Delta \hat{\xi}_{j} \Delta \hat{\xi}_{k}\right\rangle+\left\langle\Delta \hat{\xi}_{k} \Delta \hat{\xi}_{j}\right\rangle\right) .
$$


Here $\Delta \hat{\xi}_{j}=\hat{\xi}_{j}-\left\langle\hat{\xi}_{j}\right\rangle$ and $\left\langle\hat{\xi}_{j}\right\rangle=\operatorname{Tr}\left(\hat{\rho} \hat{\xi}_{j}\right)$. In our analysis of the coupler we shall assume that all processes are spontaneous, i.e., both fundamental modes are initially in the vacuum state. The covariance matrix corresponding to this input state is proportional to the identity matrix,

$$
\mathbf{V}(0)=\frac{1}{2}\left(\begin{array}{llll}
1 & 0 & 0 & 0 \\
0 & 1 & 0 & 0 \\
0 & 0 & 1 & 0 \\
0 & 0 & 0 & 1
\end{array}\right)
$$

The evolution of the covariance matrix corresponding to the symplectic transformation (9) is given by the following formula:

$$
\mathbf{V}(z)=\mathbf{S}(z) \mathbf{V}(0) \mathbf{S}^{T}(z),
$$

where $T$ stands for the transposition.

Quantum entanglement is one of the manifestations of the nonclassical features of the quantum states. The $\mathrm{CV}$ quantum state is said to have a classical analogue if its density matrix can be written as a convex mixture of projectors on coherent states. Such states cannot be entangled and they can exhibit only classical correlations. Since the nonclassicality is a necessary prerequisite for the entanglement [13] it is of interest to investigate whether and how much nonclassical are the states generated in the nonlinear coupler. This issue has been addressed in many recent papers devoted to the analysis of the quantum-statistical properties of light propagating in nonlinear couplers 8, 9]. Various kinds of nonclassical behaviour have been predicted, such as the generation of light with sub-Poissonian photon number statistics, and single-mode or two-mode squeezed light.

For Gaussian states, the nonclassicality criterion is particularly simple because these states are nonclassical if and only if (iff) they are squeezed. More formally, we say that the (generally $N$-mode) Gaussian state is nonclassical iff there exists a quadrature $\hat{X}$ that is a linear combination of the quadratures $\hat{x}_{j}$ and $\hat{p}_{j}$ of the $N$ involved modes such that the variance of $\hat{X}$ is below the coherent-state level $1 / 2$. The smallest variance obtained as minimum over the variances of all possible linear combinations of the quadratures $\hat{x}_{i}$ and $\hat{p}_{j}$ is called the generalized squeeze variance $\lambda[12$. This variance can be calculated as the lowest eigenvalue of the covariance matrix (12)

$$
\lambda(z)=\min \{\operatorname{eig}[\mathbf{V}(z)]\} .
$$

The Gaussian state is nonclassical and squeezed iff $\lambda<$ $1 / 2$. In our discussion of the coupler's behavior we distinguish two main regimes of operation. In the first regime, the linear coupling dominates over the nonlinear interactions

$$
2\left|g_{L}\right|>\left|g_{A}+g_{B}\right|
$$

and the coupler operates below the threshold. If the opposite inequality holds then the nonlinear interactions in

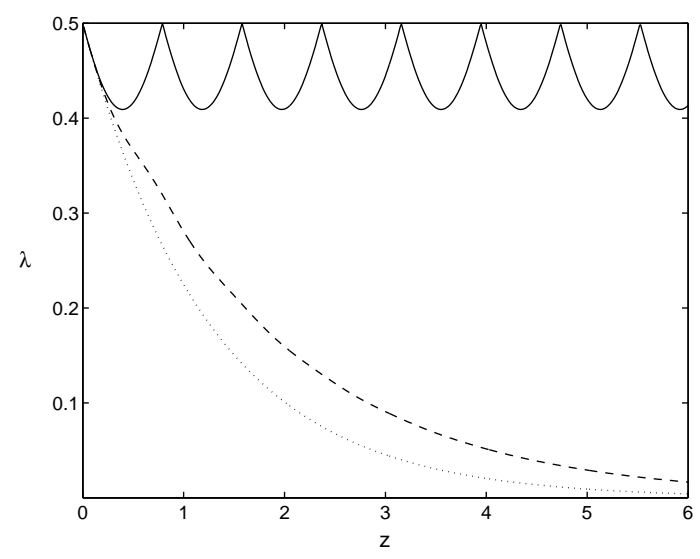

FIG. 2: The dependence of the generalized squeeze variance $\lambda$ on the length $z$ of the symmetric coupler operating below the threshold, $\left|g_{L}\right|=2,\left|g_{A}\right|=\left|g_{B}\right|=0.2$. The three curves correspond to the phase differences $\Delta \phi=0$ (solid line), $\Delta \phi=$ $\pi / 2$ (dashed line), and $\Delta \phi=\pi$ (dotted line).

the waveguides become dominant and the coupler operates above the threshold. The threshold condition $\left(2\left|g_{L}\right|=\left|g_{A}+g_{B}\right|\right)$ was determined by analyzing the eigenvalues of the matrix $\mathbf{M}$ (see also Ref. [14]). These eigenvalues are complex below the threshold, which leads to oscillatory dynamics typical for the linear coupling. Above the threshold, all eigenvalues are real and the dynamics of the coupler is reminiscent of a pure amplification process.

The behavior of the coupler also significantly depends on the phase matching condition. It can be inferred from the analytical expressions for the symplectic transformation $\mathbf{S}(z)$ that there is a single effective phase difference

$$
\Delta \phi=\phi_{A}-\phi_{B}+2 \phi_{L}
$$

that controls the dynamics of the coupler.

In figure 2 we plot the dependence of the generalized squeeze variance $\lambda$ on the length $z$ of the symmetric coupler $\left(\left|g_{A}\right|=\left|g_{B}\right|\right)$ that operates below the threshold. The three curves correspond to three different choices of the phase difference $\Delta \phi$. For $\Delta \phi=0$ we observe a periodic evolution of $\lambda(z)$. In this specific case, the eigenvalues of $\mathbf{M}$ are purely imaginary, the dynamics is purely oscillatory, and the quantum state of the two modes periodically returns to the initial vacuum state. For the two other choices $\Delta \phi=\pi / 2$ and $\Delta \phi=\pi$ the parametric amplification sets on and the squeeze variance exponentially decreases with growing length of the coupler. The squeezing is fastest for $\Delta \phi=\pi$. For comparison, we plot in figure 3 the function $\lambda(z)$ for symmetric coupler operating above the threshold. It follows that in this case the phase difference almost does not influence the dynamics of squeezing and the three curves shown in Fig. 3 corresponding to three different phase shifts $\Delta \phi$ almost coincide.

For the asymmetric coupler $\left(\left|g_{A}\right| \neq\left|g_{B}\right|\right)$ below the threshold, the eigenvalues of $\mathbf{M}$ have nonzero both imag- 


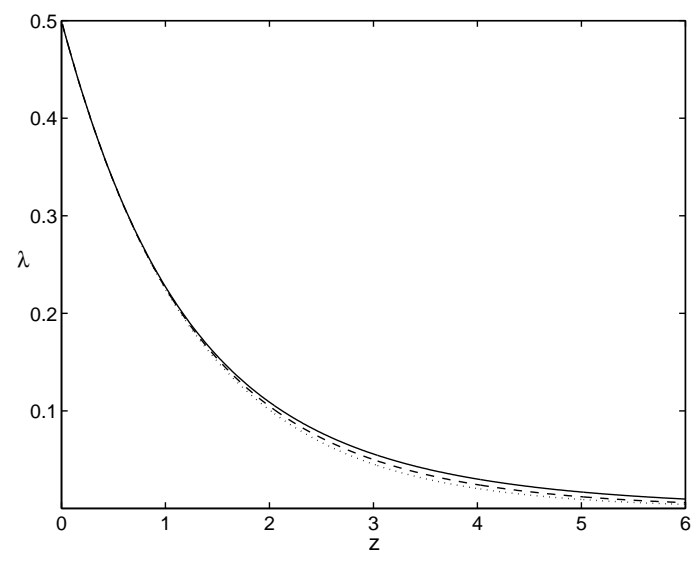

FIG. 3: The dependence of the generalized squeeze variance $\lambda$ on the length $z$ of the symmetric coupler operating above the threshold, $\left|g_{L}\right|=0.15,\left|g_{A}\right|=\left|g_{B}\right|=0.2$. The three curves correspond to the phase differences $\Delta \phi=0$ (solid line), $\Delta \phi=\pi / 2$ (dashed line), and $\Delta \phi=\pi$ (dotted line).

inary and real parts giving rise to periodically modulated exponential decrease of $\lambda(z)$. The modulations smooth out as $\Delta \phi$ goes to $\pi$. Above the threshold $\lambda(z)$ exhibits qualitatively the same behavior as in the symmetric case.

\section{SEPARABILITY AND ENTANGLEMENT}

Having discussed the squeezing of light in the coupler, we now turn our attention to the generation of entangled states. A state $\hat{\rho}_{A B}$ of two subsystems $A$ and $B$ is entangled iff $\hat{\rho}_{A B}$ cannot be written as a convex mixture of product states

$$
\hat{\rho}_{A B} \neq \sum_{j} p_{j} \hat{\rho}_{A, j} \otimes \hat{\rho}_{B, j}, \quad p_{j}>0,
$$

where $\hat{\rho}_{A, j}$ and $\hat{\rho}_{B, j}$ are states of subsystems $A$ and $B$. Since we assume that the fundamental modes are initially in pure vacuum states and the evolution is unitary, the two-mode state is pure for all $z$. It holds that every pure bipartite state $|\psi\rangle_{A B}$ that is not a product state is entangled. While it is easy to decide whether a pure state is separable or not, this problem is much more difficult for the general mixed bipartite state. Several separability criteria have been proposed in the literature. Among them, the most powerful and important is the Peres-Horodecki (PH) criterion 15, 16, 17]. According to this criterion, a quantum state $\hat{\rho}_{A B}$ is entangled if the partially transposed density matrix

$$
\left(\hat{\rho}_{A B}\right)_{m \alpha, n \beta}^{T_{A}} \equiv\left(\hat{\rho}_{A B}\right)_{n \alpha, m \beta}
$$

is nonpositive, i.e., it has at least one negative eigenvalue. In general, this is only a sufficient condition on entanglement and there exist entangled states with positive partially transposed density matrix (the so-called

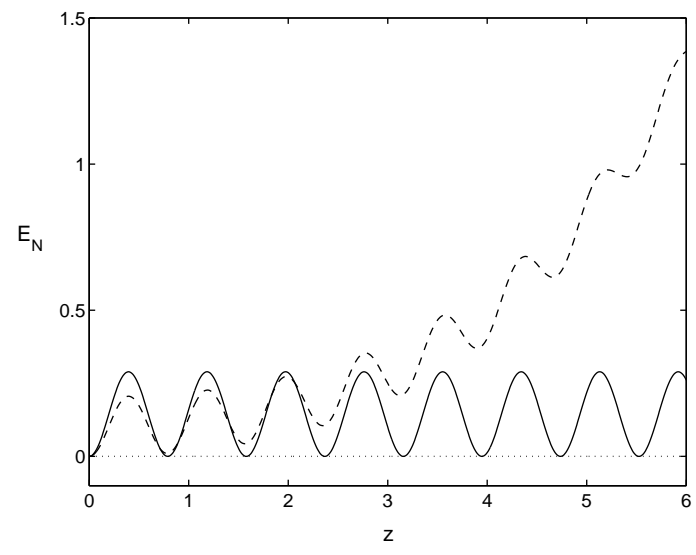

FIG. 4: The dependence of the logarithmic negativity $E_{N}$ on the length $z$ of the symmetric coupler operating below the threshold, $\left|g_{L}\right|=2,\left|g_{A}\right|=\left|g_{B}\right|=0.2$. The three curves correspond to the phase differences $\Delta \phi=0$ (solid line), $\Delta \phi=$ $\pi / 2$ (dashed line), and $\Delta \phi=\pi$ (dotted line).

bound entangled states). However, in case of two-mode bipartite Gaussian states this criterion turns out to be both sufficient and necessary [18, 19]. The PH criterion is qualitative, it tells us whether the state is entangled or separable, but it does not quantify the amount of the entanglement present in that state. For this purpose, various entanglement measures have been proposed. The $\mathrm{PH}$ criterion suggests that a function of the negative eigenvalues of the partially transposed density matrix could be a good entanglement measure. Indeed, such measure can be constructed and it is called the logarithmic negativity 10]

$$
E_{N}(\hat{\rho})=\log _{2}[1+2 \mathcal{N}(\hat{\rho})] .
$$

Here the negativity $\mathcal{N}(\hat{\rho})$ is the sum of the absolute values of the negative eigenvalues $\mu_{j}$ of the partially transposed matrix $\hat{\rho}_{A B}^{T_{A}}$

$$
\mathcal{N}(\hat{\rho})=\sum_{j}\left|\mu_{j}\right|
$$

The logarithmic negativity $E_{N}$ has the great advantage that it can be easily computed for any bipartite Gaussian state. In fact, analytical formula for $E_{N}$ has been derived for the general mixed two-mode Gaussian state. The details can be found in [10], here we only mention the main results and formulas.

It is convenient to decompose the covariance matrix as follows

$$
\mathbf{V}=\left(\begin{array}{cc}
\mathbf{A} & \mathbf{C} \\
\mathbf{C}^{T} & \mathbf{B}
\end{array}\right)
$$

where $\mathbf{A}$ and $\mathbf{B}$ are the covariance matrices of the modes on Alice's and Bob's sides, respectively, and $\mathbf{C}$ contains the intermodal correlations. We must calculate the symplectic spectrum $\left(c_{1}, c_{2}\right)$ of $\mathbf{V}$ that can be obtained by 


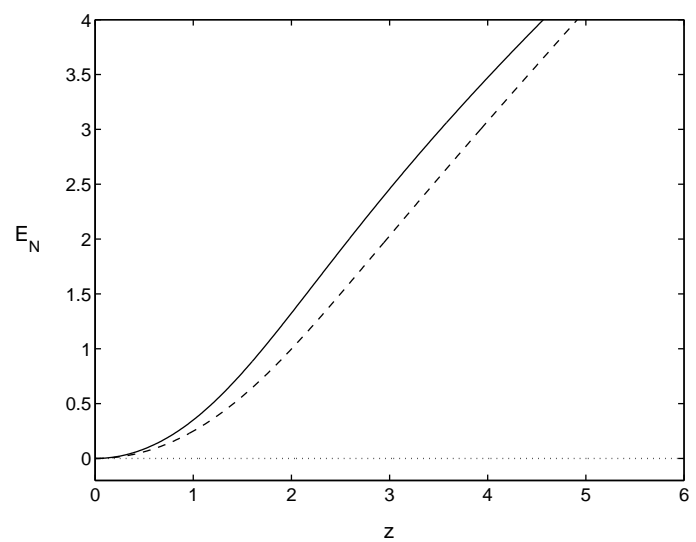

FIG. 5: The dependence of the logarithmic negativity $E_{N}$ on the length $z$ of the symmetric coupler operating above the threshold, $\left|g_{L}\right|=0.15,\left|g_{A}\right|=\left|g_{B}\right|=0.2$. The three curves correspond to the phase differences $\Delta \phi=0$ (solid line), $\Delta \phi=\pi / 2$ (dashed line), and $\Delta \phi=\pi$ (dotted line).

solving the biquadratic equation (only positive roots are considered)

$$
\zeta^{4}+(\operatorname{det} \mathbf{A}+\operatorname{det} \mathbf{B}-2 \operatorname{det} \mathbf{C}) \zeta^{2}+\operatorname{det} \mathbf{V}=0 .
$$

The logarithmic negativity is a function of the symplectic eigenvalues

$$
E_{N}=\sum_{j=1}^{2} F\left(c_{j}\right)
$$

where the function $F(c)$ reads

$$
F(c)=\left\{\begin{array}{cc}
0 & \text { for } 2 c \geq 1 \\
-\log _{2}(2 c) & \text { for } 2 c<1
\end{array}\right.
$$

The evolution of the logarithmic negativity is plotted in Fig. 4. The parameters are the same as in Fig. 2, so the coupler is below the threshold. As could have been expected, for $\Delta \phi=0$ we get a purely oscillatory evolution and $E_{N}$ periodically attains its maximum. The oscillations are clearly present also for $\Delta \phi=\pi / 2$ but the general trend is that $E_{N}$ grows with $z$. This is in agreement with the fact that for this detuning the state becomes more and more squeezed with growing $z$, cf. Fig. 2. Remarkably, $E_{N}(z)=0$ for all $z$ when $\Delta \phi=\pi$. For this particular phase difference, the coupler behaves like two decoupled single mode squeezers that do not interact at all [14]. Figure 5 shows the behavior of $E_{N}(z)$ when the coupler operates above the threshold. We can see that the oscillatory evolution is replaced with a monotonic growth of $E_{N}(z)$ with $z$. However, the amount of entanglement significantly depends on $\Delta \phi$ even when the coupler is above the threshold. This is in marked difference with the generalized squeeze variance that is to a large extent insensitive to the choice of $\Delta \phi$, see Fig. 3.

In the case of the asymmetric coupler above the threshold $E_{N}$ grows monotonously with growing $z$. For small
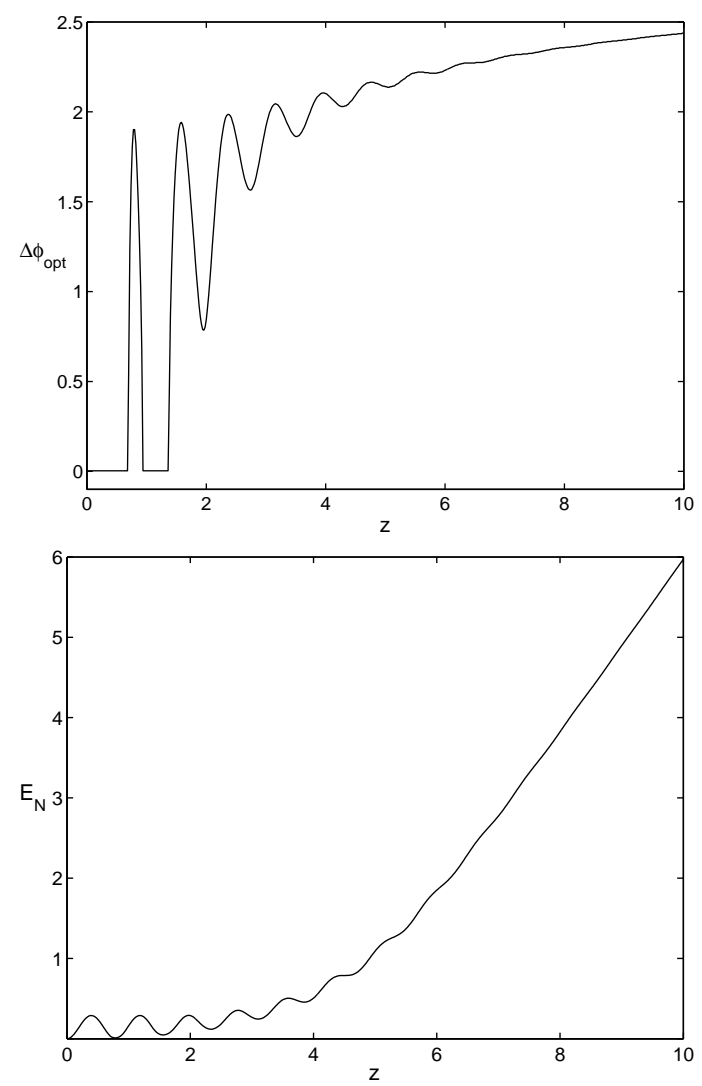

FIG. 6: The dependence of the optimal phase difference $\Delta \phi_{\text {opt }}$ and the maximum of the logarithmic negativity $E_{N}$ on the length $z$ of the symmetric coupler operating below the threshold $\left|g_{L}\right|=2,\left|g_{A}\right|=\left|g_{B}\right|=0.2$.

$z$ the rapidity of the growth decreases as $\Delta \phi$ goes from 0 to $\pi$. Below the threshold $E_{N}$ behaves similarly as in the symmetric case. The only difference occurs for $\Delta \phi=\pi$ when $E_{N}$ changes periodically and no decoupling of the waveguides takes place.

The results depicted in Figs. 4 and 5 indicate that for each $z$ and a given set of parameters, we may tune the phase difference such as to maximize the entanglement. We have performed numerical calculations and determined the optimal phase difference $\Delta \phi_{\mathrm{opt}}$ and the corresponding maximal achievable $E_{N}$. The results for the coupler operating below the threshold are presented in Fig. 6. The $\Delta \phi_{\text {opt }}$ exhibits an interesting behavior. Initially, it is optimal to set $\Delta \phi_{\text {opt }}=0$ and this is optimal up to certain length $z_{0}$. At this distance, the optimal phase difference becomes to deviate from 0 and $\Delta \phi_{\text {opt }}$ oscillates with $z$. The figure 6 suggests that for large $z$, $\Delta \phi_{\text {opt }}$ approaches some fixed asymptotic value. We note that similar results were obtained also for the coupler above the threshold. 


\section{CONCLUSIONS}

In this paper we have explored the controllable compact source of squeezing and entanglement formed by a nonlinear optical coupler composed of two nonlinear waveguides operating by means of spontaneous degenerate parametric downconversion. Firstly, the behavior of the generalized squeeze variance in the below the threshold and above the threshold regimes has been analyzed. Secondly, by calculating the logarithmic negativity the possibility of the entanglement generation in the coupler has been demonstrated. In particular, it was shown that the amount of the entanglement can be controlled via the effective phase difference (15). Finally, with the help of the numerical calculation the optimal effective phase difference providing maximum entanglement at the output of the coupler has been found. Our theoretical analysis thus clearly illustrates the potential utility of the coupler for controlled generation of CV entanglement.

\section{ACKNOWLEDGMENTS}

This work was supported by Project LN00A015 and Research Project No. CEZ: J14/98 of the Czech Ministry of Education and by the EU grant under QIPC Project IST-1999-13071 (QUICOV).
[1] A. Einstein, B. Podolsky, and N. Rosen, Phys. Rev. 47, 777 (1935).

[2] C. H. Bennett, G. Brassard, C. Crepeau, R. Jozsa, A. Peres, and W. K. Wootters, Phys. Rev. Lett. 70, 1895 (1993).

[3] M. Zukowski, A. Zeilinger, M. A. Horne, and A. K. Ekert, Phys. Rev. Lett. 71, 4287 (1993); J.-W. Pan, D. Bouwmeester, H. Weinfurter, and A. Zeilinger, Phys. Rev. Lett. 80, 3891 (1998).

[4] C. H. Bennett and S. J. Wiesner, Phys. Rev. Lett. 69, 2881 (1992).

[5] A. K. Ekert, Phys. Rev. Lett. 67, 661 (1991).

[6] A. Barenco, D. Deutsch, A. Ekert and R. Jozsa, Phys. Rev. Lett. 744083 (1995).

[7] A. Furusawa, J. L. Sørensen, S. L. Braunstein, C. A. Fuchs, H. J. Kimble, and E. S. Polzik, Science 282, 706 (1998).

[8] J. Peřina, Jr., and J. Peřina, Progress in Optics, E. Wolf (Ed.), Elsevier, Amsterdam, 2000, p. 361.

[9] J. Fiurášek, and J. Peřina, Coherence and Statistics of
Photons and Atoms, J. Peřina (Ed.), J. Wiley, New York, 2001, p. 65.

[10] G. Vidal, R. F. Werner, Phys. Rev. A 65, 032314 (2002).

[11] J. Peřina, and J. Peřina Jr., J. Mod. Optics 43, 1951 (1996).

[12] R. Simon, N. Mukunda, and B. Dutta, Phys. Rev. A 49, 1567 (1994).

[13] M. S. Kim, W. Son, V. Bužek, and P. L. Knight, Phys. Rev. A 65, 032323 (2002).

[14] J. Fiurášek, and J. Peřina, Phys. Rev. A 62, 033808 (2000).

[15] R. Horodecki, P. Horodecki, and M. Horodecki, Phys. Lett. A 200, 340 (1995).

[16] A. Peres, Phys. Rev. Lett. 77, 1413 (1996).

[17] M. Horodecki, P. Horodecki, and R. Horodecki, Phys. Lett. A 223, 1 (1996).

[18] L.-M. Duan, G. Giedke, J.I. Cirac, and P. Zoller, Phys. Rev. Lett. 84, 2722 (2000).

[19] R. Simon, Phys. Rev. Lett. 84, 2726 (2000). 